\title{
Designing complex optical filters using photonic crystal microcavities
}

Mohammad Soltani, Amil Haque, Babak Momeni, Ali Adibi, Yong Xu, et al.

Mohammad Soltani, Amil Haque, Babak Momeni, Ali Adibi, Yong Xu, Reginald K. Lee, "Designing complex optical filters using photonic crystal microcavities," Proc. SPIE 5000, Photonic Crystal Materials and Devices, (9 July 2003); doi: 10.1117/12.480062

SPIE Event: Integrated Optoelectronics Devices, 2003, San Jose, CA, United States 


\title{
Designing Complex Optical Filters Using Photonic Crystal Microcavities
}

\author{
Mohammad Soltani, Amil Haque, Babak Momeni, Ali Adibi \\ School of Electrical and Computer Engineering, Georgia Institute of Technology
}

Yong Xu, Reginald. K. Lee

Department of Applied Physics, California Institute of Technology

\begin{abstract}
By adding a point defect to a photonic crystal structure, a microcavity can be made to trap electromagnetic energy with wavelength inside the photonic bandgap (PBG). This property together with other unique properties of photonic crystals enables us to control the propagation and spectrum of transmitted wave inside the photonic crystal waveguiding structures and to design and implement microscale optical filters. In this paper we focus on the design of notch filters in photonic crystal waveguides based on the coupling of waveguide and cavity. We discuss about the properties of a single cavity and its necessary modifications to achieve efficient coupling between cavity and waveguide and eventually obtain desired notch filters at the frequency range of interest. We also discuss the coupling of multi-cavities to a waveguide and the possibility of attaining filters with better performances is presented, and the spectrum and lineshapes of the resulting filters are characterized.
\end{abstract}

Keywords: Photonic Crystal, Cavity, Waveguide, Filter

\section{INTRODUCTION}

Photonic crystals [1,2] have inspired a lot of research recently due to their ability to control the propagation of light. The periodic variation of the permittivity in photonic crystals can resort in a photonic bandgap [3-5], i.e., a range of frequencies with no allowed electromagnetic mode. By creating a defect in these structures, the periodicity and consequently the completeness of the bandgap are broken and light can be localized at the defect region with the frequency corresponding to the defect frequency inside the gap. Two possible defect types are point defect and line defect which correspond to photonic crystal cavities [3] and photonic crystal waveguides [3], respectively. The properties of photonic crystal cavities have been extensively analyzed [6-8] and experimentally demonstrated $[9,10]$. Cavities with theoretical quality factors (Q) more than $20000[11,12]$ have been designed. Experimental values of as high as $\mathrm{Q}=4000$, have been demonstrated for photonic crystal cavities [13].

Photonic crystal cavities with high Q can be effectively used for point defect lasers [14], optical filters [15-18], and cavity quantum electrodynamics (QED) [19]. These cavities have been used for designing add/drop filters [18] for wavelength division multiplexing (WDM) applications. Also, initial demonstrations show the possibility of using these cavities for separating optical signals with different wavelengths in the filter characteristics (i.e., the variation of the filter output with wavelength) of the simple point defect cavities are not flat over the WDM channel. The design of photonic crystal cavities with prescribed frequency response is highly needed for several applications. In this paper, we focus on designing such filters by altering the geometry defects and by using multiple cavities. While we concentrate on notch filters in this paper, the results can be applied to other types of filters.

\section{PHOTONIC CRYSTAL FILTERS BASED on WACEGUIDE CAVITY COUPLING}

Figures 1.a and 1.b show two typical point defects in triangular lattice photonic crystal of air columns in dielectric host. As shown in Figure 1.a, by increasing the radius of an air hole, a cavity known as acceptor type is created. The resonance frequency of this cavity is pushed up from the lower band of the PBG. In Figure 1.b by decreasing the radius of a hole (in this case, the radius is zero) the cavity is generated. The resonance frequency of this cavity, known as donor type is pulled down from the upper band of the PBG. As shown in Figure 1.b, this cavity is degenerate and supports two different modes corresponding to the same frequency, and Figure 1.c shows the spectrum of this cavity. Generally, acceptor and donor type cavities have different symmetry properties, different field profiles, different field orientations and concentrations. Due to the symmetry of the triangular lattice photonic crystals, the electromagnetic modes of the cavities in Figure 1 have 60 degree rotation symmetries. 
In Figure 2.a the typical structure of a line defect which acts as a waveguide is shown. It supports two modes one with even profile and the other one with odd profile with respect to the propagation direction. Figure 2.b shows the dispersions of these modes which are inside the PBG.

By putting a cavity next to a waveguide, it can be coupled to the waveguide at its resonance frequency to trap some of the electromagnetic energy propagating in the waveguide. This new structure can modify the transmission and dispersion of propagating modes of the waveguide so that by proper coupling, the dropping of wave with specific frequency close to the resonance frequency of the cavity is possible. To drop a signal from a waveguide, two main architectures have been proposed. The first case is to drop a signal from the waveguide to a cavity and send it vertically outside the structure [18]. The second case is to drop the signal by a cavity or multiple cavities and couple it back to the same waveguide (reflection) or send it to another waveguide [15-17, 20]. Figure 3 show three possible architectures relevant to the second case. In Figure 3.a an array of cavities is coupled to the waveguide and the resulting structure acts as a reflection band stop (or notch) filter. The structure in Figure 3.b performs the same operation, but in this case cavities are present at both sides of the waveguide. Finally Figure 3.c shows the dropping of signal from one waveguide by the cavity and sending it to another waveguide. In this case by proper design of the cavity, the ratio of the transmission to the backward and forward direction, in the second waveguide can be controlled.

Practically, single mode photonic crystal waveguides are the case of interest, therefore the main requirement in the design of the cavity for efficiently coupling it to the waveguide, is to put its resonance frequency at the single mode operation frequency range of the waveguides. As shown in Figure 2.b the guiding structure supports single mode operation at two separate ranges of frequencies. These ranges are below and above the region in which waveguide has two modes. As mentioned, we can design two classes of cavities, which are donors and acceptors. But according to Figure 1.a the energy concentration in the acceptor case is mostly in the air holes while that of the donor type is mostly in the dielectric. Thus, the acceptor type cavities are appropriate for out-of-plane coupling of the filtered (or dropped) signal, and donor type cavities are preferable for in-plane filtering. The latter case is more relevant in integrated photonic circuits. Thus we focus only on the donor type cavities in the rest of this paper. In designing such donor type cavities, the resonance frequency of the cavity must be in one of the two frequency regions in which the waveguide is single mode (see Figure 2.b). Due to the different properties of waveguide and cavity at these two ranges of frequencies, the performance of filtering may be different and we need to consider these different properties. Other important considerations to obtain efficient waveguide to cavity coupling are: 1- The separation distance between the cavity and waveguide. 2- Field profile of the cavity and its orientation with respect to the field profile of the waveguide, and 3Number of the degenerate modes of the cavity at a single resonance frequency.

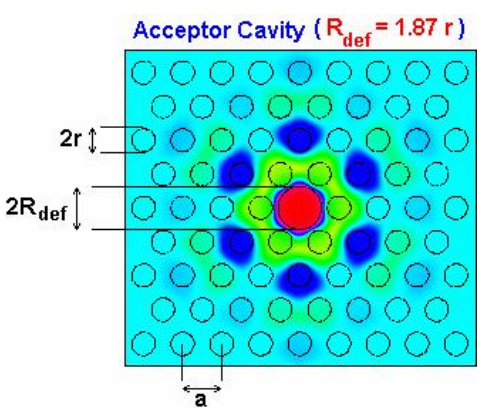

(a)

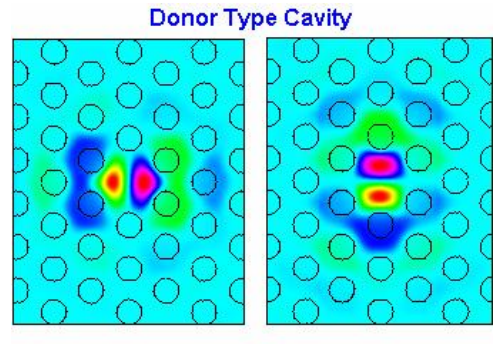

(b)

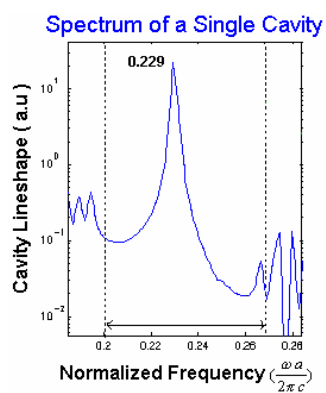

(c)

Fig. 1: (a) Structure and the TM field profile of an acceptor type point defect made by increasing the radius of the central hole, (b) and (c) The degenerate TM field profiles and spectra of the modes of a donor type cavity made by removing one air hole. The radii of all other holes are $\mathrm{r}=0.3 \mathrm{a}$. The dielectric host is GaAs.

\section{SIMULATIONS}

The main structure we used in the analysis of this paper is a two dimensional (2-D) triangular lattice photonic crystal of air columns in GaAs. The relative permittivity of GaAs is 12.96 , and the radii of the air columns are $30 \%$ of the lattice constant unless otherwise stated. The polarization of light, throughout this paper, is TM (magnetic field perpendicular to plane of periodicity) to assure the largest photonic bandgap. We used the method of finite difference time domain (FDTD) [22] to get the spectrum of point defects as shown in Figure 1. A perfectly matched layer (PML) [23] is put 
around the structure containing point defect at all boundaries. To obtain the dispersion diagram of the guided modes of a photonic crystal waveguide, also called PBG waveguide, which is created by removing a row of air columns in the $\Gamma-\mathrm{K}$ direction, we used the method of plain waves expansion (PWE) [3]. To analyze the coupling of waveguide and cavity the electromagnetic mode (by a Huygens source [24]) is launched from a conventional slab to PBG waveguide which is coupled to the cavity(s) with PML at all boundaries. The power transmission spectra are calculated by taking the Fourier transform of the fields calculated by FDTD and then integrating the Poynting vector over the surface of 60 calculation cells centered at the middle of the PBG waveguide. Further details of this method can be found in [21]

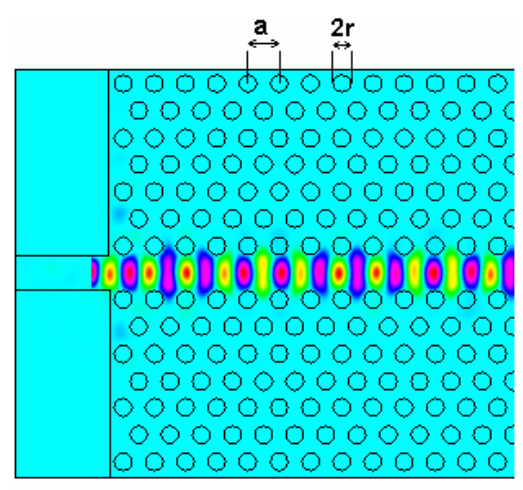

(a)

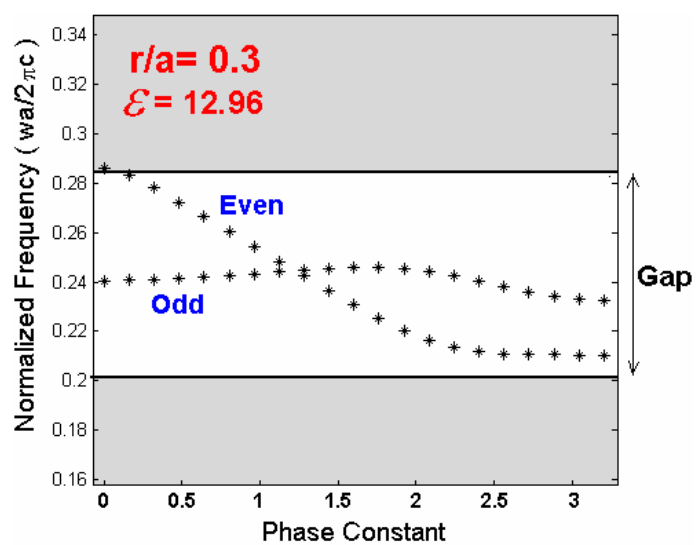

(b)

Fig. 2: (a) Structure of line defect (waveguide) and the field profile of the fundamental TM mode in a triangular lattice photonic crystal of air columns in GaAs, and (b) the dispersion diagram of the guided modes inside the PBG in the $\Gamma-\mathrm{K}$ direction.

(a)

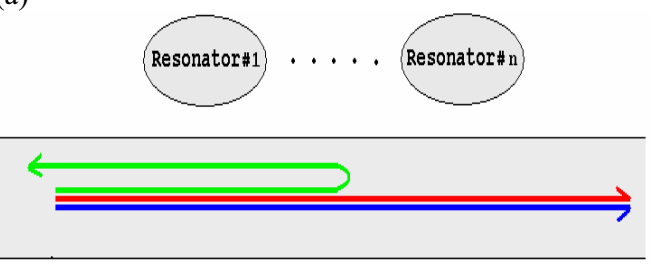

(b)

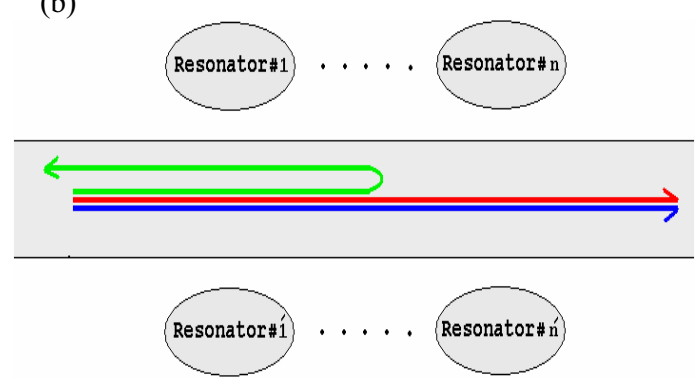

(c)

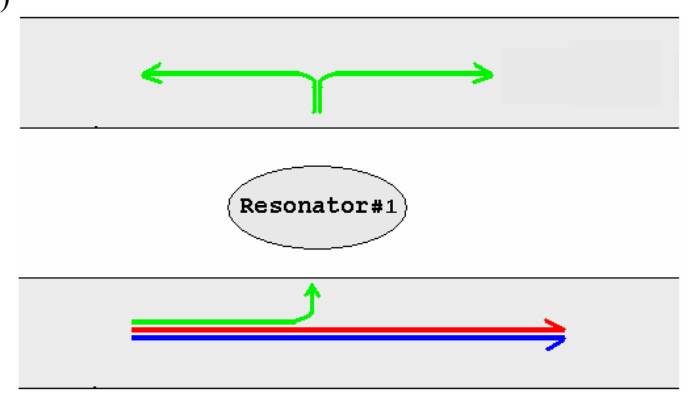

Fig. 3: Three different architectures for in-plane channel drop filtering. (a) An array of resonators side- coupled to a waveguide to reflect the dropped signal, (b) an array of double side resonators coupled to the waveguide, and (c) two waveguide coupled to each other through a resonator. 


\section{DESIGN of PHOTONIC CRYSTAL CAVITIES for FILTERS}

In this section, discuss the properties of photonic crystal cavity structures that can be used for designing in-plane notch filters. As discussed in section 2, the resonance frequency of the cavity can be chosen in one of the two frequency regions for single mode waveguide operation.

First, we focus on the design of filter at the single mode region which is close to upper band in Figure 2.b. Figure 4.a shows the structure of a waveguide coupled to two defects at both sides of the waveguide, (or a double side defect). The defects have been created by decreasing the radius of the air holes at the origin of each defect region. Figure 4.b shows the transmission of the single side cavity-waveguide structure for different sizes of the defect when one of the defects in Figure 4.a is absent. Figure 4.b shows that for the larger sizes of the defect the reflection at the resonance frequency of the cavity is higher. This is because the higher frequencies are closer to the upper band and the field profiles of the cavity modes, at their resonance frequencies, become more extended than lower frequencies. So, the mode volume of these modes is larger and therefore, they have stronger coupling to the field profile of the waveguide. Figure 4.c compares the transmission of a double side and a single side couple defect. As shown in the figure, the reflection at the notch frequency for the double side structure is stronger than that for the single side structure. Also the frequency of the filter in the double side case is lower than that in the single side case.

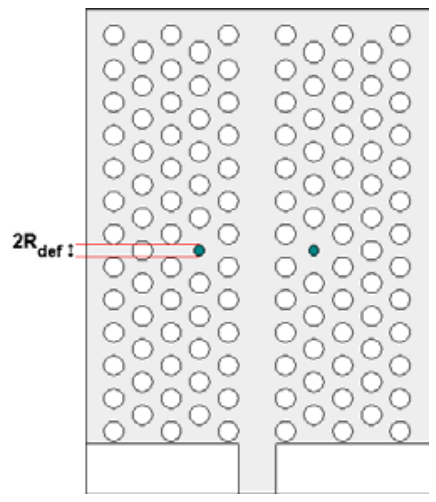

(a)

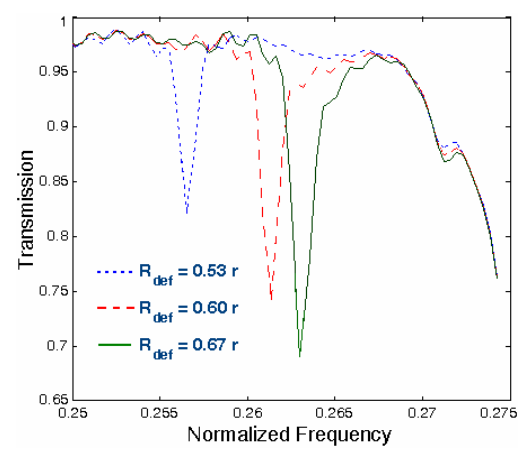

(b)

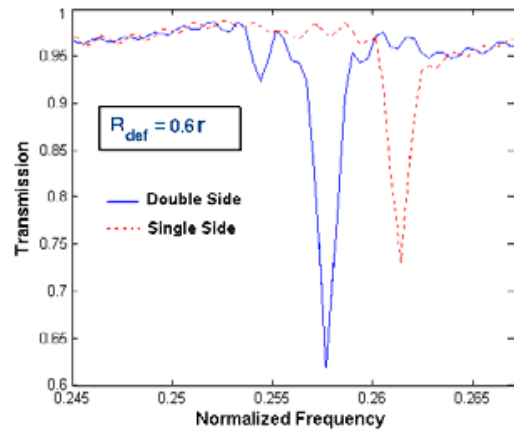

(c)

Fig. 4: (a) Structure of a PBG waveguide side coupled to a pair of defects, the defects are air columns with sizes smaller than the sizes of original holes, (b) power transmission spectra of the structure, when only one of the two defect is present (single side defect), for different sizes of the defect, and (c) power transmission spectra, when both the defects are present (double side defect), for different sizes of the defects.

The presence of two similar defects at both sides of the waveguide results in stronger coupling and larger reflection. Meanwhile, these two cavities are coupled to each other through the waveguide, therefore, the resonance frequencies of the coupled structure is different from that of a single cavity (corresponding to the single side case). This explains the difference between the filtering frequency of the single side and double side structure.

An important issue in the design of a notch filter is to precisely control the center frequency of the filter. To achieve this goal, we need to change the geometry of the holes in the cavity region. One way is to change the radius of the defect hole to control the center frequency of the cavity. But as Figure 4.b shows, the variation of the center frequency using this method is very large and besides, the reflection coefficient dramatically changes with changing the radius of the defect hole. Another method is to add another material inside the defect hole or at the holes next to the defect and control the transmission properties of the filter by changing the dielectric constant of this material. While interesting, filling the air holes of a planar structure with a material different from the host material is challenging. As we will show, an efficient way to modify the transmission of the filter is modify the geometry of holes surrounding the cavity to slightly change the center frequency of the defect with minimal changes to the reflection at the center frequency.

Figure 5.a shows the structure of a perturbed cavity in which the radii of two holes next to the removed hole have been changed. As mentioned in section 2, the unperturbed cavity is intrinsically degenerate and supports two modes with even and odd symmetry of the field profile. By comparing the field profile of the cavity in figure 5.a with that of the fundamental mode of a horizontal photonic crystal waveguide we can conclude that the even mode of the cavity has a 
stronger coupling to the waveguide mode. Furthermore, two of the six holes surrounding the defect region are in less contact with the even field profile while they are highly in contact with the odd field profile. Therefore, we can break the mode degeneracy by modifying the sizes of these holes, as shown in Figure 5.a. As shown in Figure 5.b, the variation of the resonance frequency of the two cavity modes with the radii of the two holes $\left(\mathrm{R}_{\mathrm{d}}\right)$ next to the defect air hole shown in Figure 5.a. As expected, the frequency of the odd modes is strongly affected (due to the large energy extent of this mode at the location of the two holes while that of the even mode is only slightly modified by changing the radii of the two air holes in a large range. Using this technique, we have obtained two goals simultaneously. The first is the design of single mode cavity and keeping the desired field profile for stronger coupling, and the second is the fine variations of the center frequency of the defect corresponding to this field profile. Besides, the even field profile is only slightly modified with these modifications, which results in only a small change in the coupling coefficient between the waveguide and the cavity.

Figure 6.a shows the structure of a double side waveguide-cavity structure after these modifications. In each cavity the radius of the central air hole is reduced while the radii of the two holes surrounding the cavity have been increased. Figure 6.b shows the transmission of the structure for different radii of the modified holes $\left(\mathrm{R}_{1}\right)$ with a fixed radius for the center hole $\left(\mathrm{R}_{\text {def }}\right)$. As we predicted, for different modifications, the center frequency of the notch filter has slight variations and also the reflection of the filter remains nearly constant.

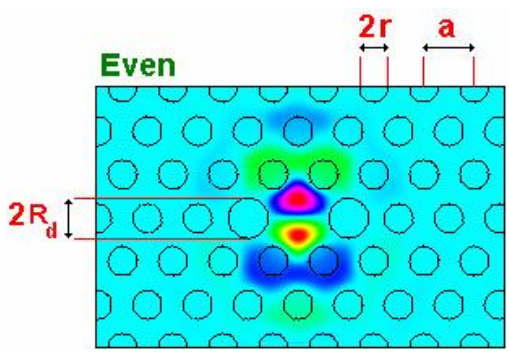

(a)

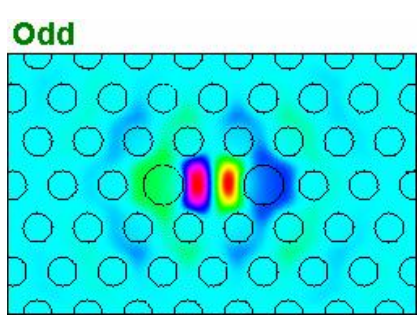

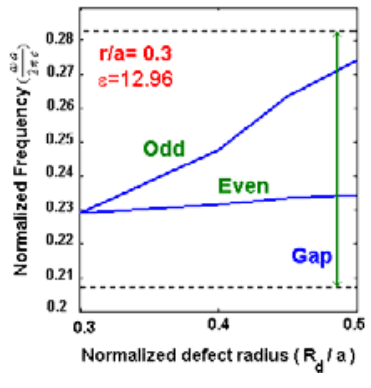

(b)

Fig. 5: (a) Structure of a modified cavity ant its field profiles, (b) variation of the resonance frequencies of the cavity modes vs. the radius of the modified holes, the radii of all other holes are $r=0.3 \mathrm{a}$.

The next step to improve the filtering response of the structure is increasing the reflection at the notch frequency. As we know from the microwave and electronic filters, by chaining an array of resonators the larger attenuations in the transmission characteristic is possible [25]. Using this idea, we propose the structure shown in Figure 7.a. where an array of defects is created at both sides or one side of the waveguide (double side vs. single side structure). Figure 7.b shows the transmission of a structure composed of an array of four double side defects coupled to the waveguide. In this figure, the transmissions for three different cases are shown: In the first case, the radii of the surrounding holes are not modified $\left(\mathrm{R}_{1}=\mathrm{r}\right)$, while in the other two cases all the radii of the two extra holes, in each cavity, have been changed. Comparing Figure 7.b with the filter response of a double side defects shown in Figures 4 and 6, we can see that using multiple cavities results in a larger reflection (better filter response). Also as we can see in Figure 7.b, by modifying the sizes of the two holes discussed previously, the fine tuning of the center frequency of the filter is possible. Figure 7.b also shows that the reflection for larger sizes of the modified holes is stronger. The reason is as follows: increasing the sizes of the modified holes which are parallel to the waveguide decreases the filed extension of the desired mode of the cavity in this direction while it increases the filed extension in the direction normal to the waveguide. This vertical extension means more overlap between the filed profiles of the waveguide and cavity. This results in stronger waveguide-cavity coupling and consequently larger reflection.

Figure 7.c shows the transmission response of modified double side defect structure for different number of defect pairs. The radius of the air column defect is $60 \%$ of the radius of the original holes $\left(\mathrm{R}_{\mathrm{def}}=0.6 \mathrm{r}\right)$, and the radii of the modified holes are $33 \%$ larger than that of the original ones $\left(\mathrm{R}_{1}=1.33 \mathrm{r}\right)$. As shown in Figure 7.c, for six double defects, large reflection is obtained. Meanwhile, the center frequency of the filter has slight variations for different number of defect pairs due to the mutual coupling of these cavities. 


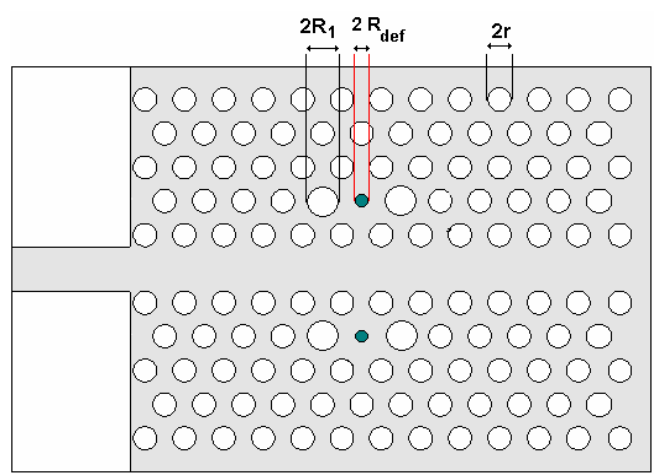

(a)

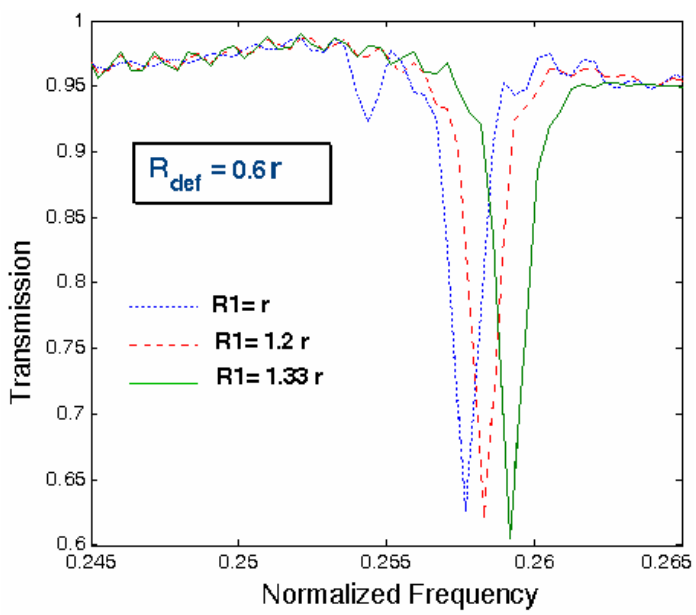

(b)

Fig. 6: (a) Structure of a PBG waveguide side coupled to a double side defects, the radius of the central hole is $60 \%$ of the original holes $\left(R_{\text {def }}=0.6 r\right)$. By changing the radii of two holes surrounding the defect $\left(R_{1}\right)$, the spectrum of the defects have been modified, $(b)$ power transmission spectra of the structure for different sizes of the modified holes $\left(\mathrm{R}_{1}\right)$.

Now, we focus on the design of notch filter at the second range of single mode operation. This range is below the range in which the waveguide supports both even and odd modes. To design a notch filter by donor type defects, the resonance frequency of these defects should be brought to this range of frequency. To achieve this, we need to increase the mode volume of the cavity to shift its resonance frequency to lower frequencies. The technique we use is decreasing the sizes of the proper holes surrounding the cavity. Figure 8.a shows the structure and field profiles of the proposed cavity, and Figure 8.b shows its spectrum. As Figure 8 shows, the sizes of four holes have been decreased and this results in larger cavity volume. It turns out that the degeneracy is broken, and the resonance frequencies of the two modes are separated and also shifted to lower frequencies. As we see these four modified holes are more in contact with the even field profile, so we expect larger change in the resonance frequency of the even mode. Comparing the spectrum of Figure 8.b with the spectrum in Figure 1.c, which is for the original cavity without modifying the radii of four surrounding holes, shows this fact. More evidently, between the two field profiles of Figure 8.a, the even one is the desired one, because it better couples to waveguide and it gives rise to stronger reflection. Also the spectrum of the even mode is at the frequency range of interest which is the lower single mode wavguiding region (see Figure 2.b). For larger separation between the resonance frequencies of these modes, we can apply the technique that we used in Figure 5. Similar to that case if we increase the radii of two holes in Figure 8, the effective mode volume of the odd field profile becomes smaller while that of the even field profile remains almost intact. This modification pushes the resonance frequency of the odd to higher frequencies. As we know, larger cavity volume results in larger field extension and larger overlapping with the field profile of the waveguide. But, in addition to designing a notch filter, we must preserve the dispersion properties of the waveguide. In fact if we put a cavity with large volume very close to the waveguide, the dispersion properties of the waveguide are highly affected. Hence, we have to put the cavity at proper distance with respect to the waveguide so that we keep both advantages of strong coupling and less distortion in the dispersion of the waveguide.

Figure 9.a shows the filter structure made by coupling two pairs of defects side coupled to a waveguide in the second range of single mode operation. As shown in this figure, the radii of holes surrounding the point defects are modified. Fig 9.b shows the transmission of the filter for two cases. In one case one defect pair and in the second case two defect pairs are coupled to the waveguide. As expected, stronger reflection and lower notch frequency are obtained for the structure with two defect pairs. As mentioned, these defects are coupled to each other through the waveguide and therefore their resonance frequency become smaller compared to the single side defect.

Therefore to design the filter at a specific frequency region with large number of defect pairs, we have to adjust the sizes of the proper holes to control the resonance frequency of the resulting filter. The technique we have used to do our simulations is to couple light from a conventional slab waveguide, as shown in Figure 9.a, to the PBG waveguide. Generally, the transmission form the slab to the PBG waveguide at this range of frequencies is lower compared to the first frequency range for single mode operation of the waveguide. Therefore, when we design the notch filter at this 
range, we have to be careful to consider both the hole size adjustments and the number of defect pairs to obtain the desired filter response with strong reflection, and keep its center frequency at region in which the transmission of light from output to the PBG waveguide or vise versa is high enough.

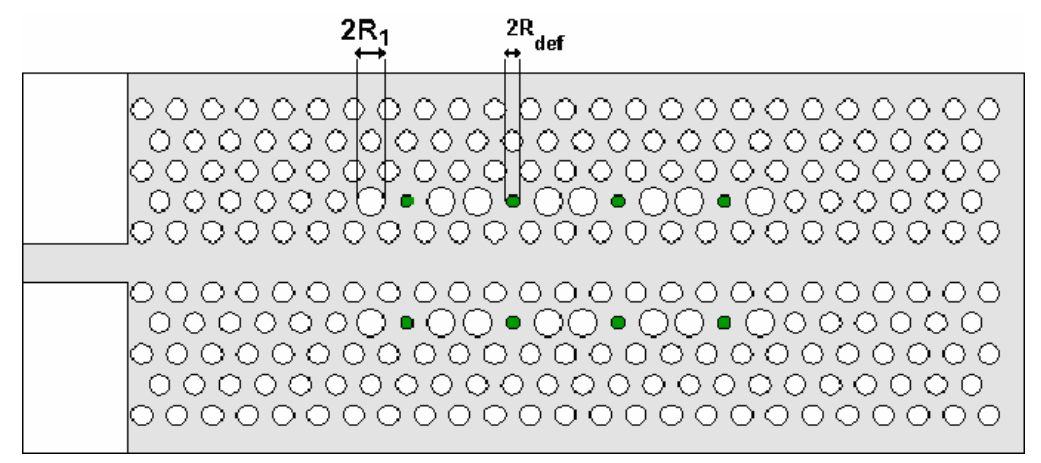

(a)

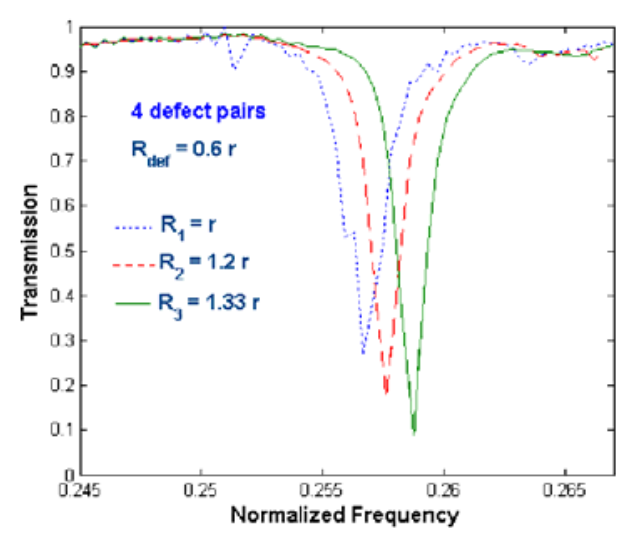

(b)

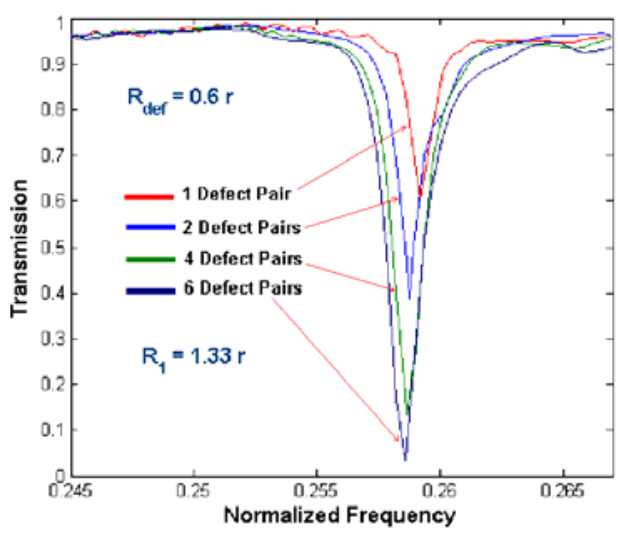

(c)

Fig. 7: Structure of a PBG waveguide coupled to an array of double side defect; the defect have been created by reducing the radius of the central hole in each defect region to $60 \%$ of the original radius $\left(\mathrm{R}_{\mathrm{def}}=0.6 \mathrm{r}\right)$, (b) power transmission spectrum of the structure in part (a) with 4 defect pairs for different sizes of the two modified holes in each defect, and (c) power transmission spectrum of the structure in part (a) with $\mathrm{R}_{1}=1.33 \mathrm{r}$ for different number of defect pairs.

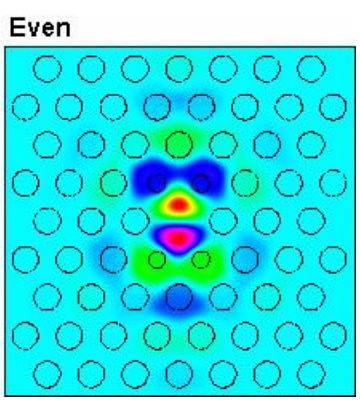

(a)
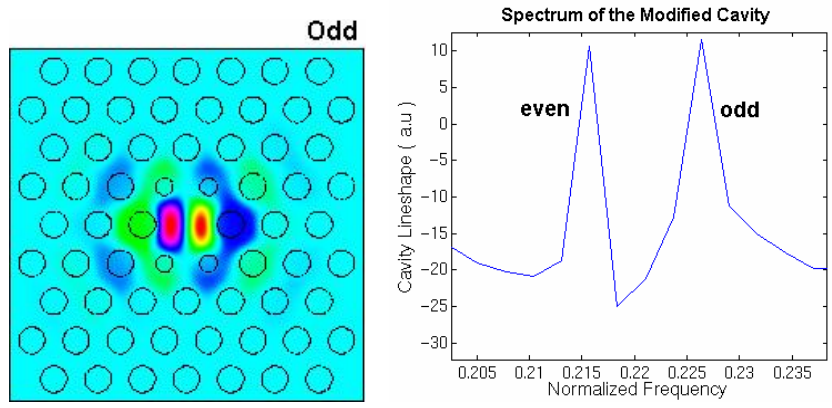

(b)

Fig. 8: (a) Structure of modified cavity with its field profiles: the cavity has been created by removing the central hole and reducing the radii of four holes surrounding the cavity to $60 \%$ of the original radius, (b) spectrum of the cavity: due to breaking the degeneracy, the spectra of the two modes are separated. 


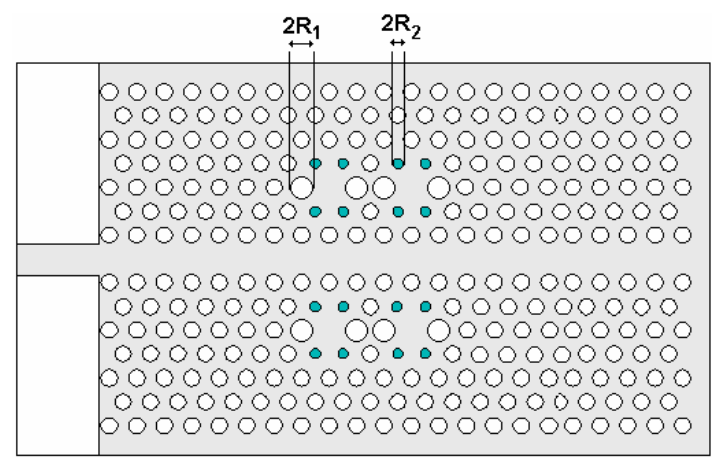

(a)

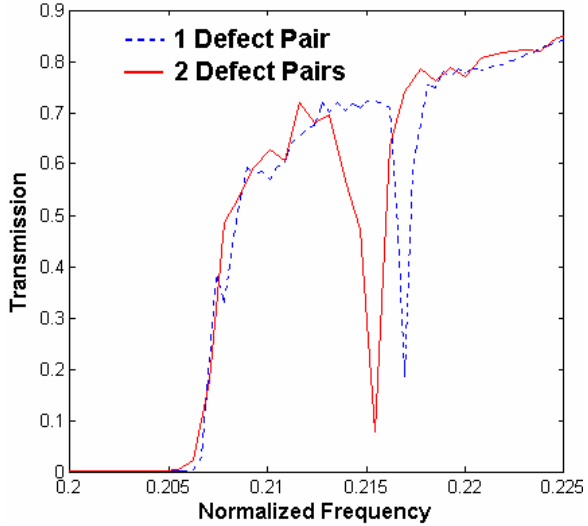

(b)

Fig. 9: (a) Structure of a waveguide coupled to arrays of double side defects: the defects are donor type and have been created by removing one air hole and modifying the radii of the surrounding holes. The mode volume of each defect is controlled by the sizes of the hole surrounding the cavity. The resonance frequency of each defect is below the odd mode operation range of the waveguide, (b) power transmission spectra of the structure in (a) for one and two double side defects. The radii of the larger and smaller holes in part (a) used for this simulation are $R_{1}=1.33 \mathrm{r}$ and $R_{2}=0.6 \mathrm{r}$ respectively.

\section{SUMMARY}

In this paper we dealt with the design of in-plane notch filters based on the side coupling of waveguide and an array of defects inside the photonic crystal structures. We discussed about the properties of a single cavity and its necessary modifications to achieve efficient coupling between cavity and waveguide and eventually obtain desired notch filters at the frequency range of interest. We showed that by modifying the geometry of air holes surrounding the original point defect, we can obtain single mode cavities with better control on their resonance frequencies. We also showed that by using multiple cavities coupled to a waveguide, filters with better performance can be obtained.

\section{REFERENCES}

1. E. Yablonovitch, "Inhibited Spontaneous Emission in Solid-State Physics and Electronics," Phys. Rev. Lett. 58, pp. 2059-2062, 1987.

2. S. John, "Strong localization of photons in certain disordered dielectric superlattices," Phys. Rev. Lett. 58, pp. 24862489, 1987.

3. J. D. Joannopoulos, R. D. Meade, J. N. Winn, Photonic Crystals, Princeton Univ. Press, 1995.

4. P. R. Villeneuve and M. Piche, "Photonic Band Gaps in Two-Dimensional Square and Hexagonal Lattices," Phys. Rev. B 46, pp. 4969-4972, 1992.

5. Y. Xu, H. B. Sun, J. Y. Ye, S. Matsuo, H. Misawa, "Fabrication and direct transmission measurement of high-aspectratio two-dimensional silicon-based photonic crystal chips," J. Opt. Soc. Am. B 18, pp.1084-1091, 2001.

6. P. R. Villeneuve, S. Fan, and J. D. Joannopoulos, "Microcavities in photonic crystals: Mode symmetry, tunability, and coupling efficiency," Phys. Rev. B 54, pp. 7837-7842, 1996.

7. O. Painter, J. Vuc;kovic, and A. Scherer, "Defect modes of a two-dimensional photonic crystal in an optically thin dielectric slab," J. Opt. Soc. Am. B 16, pp. 275-285, 1999.

8. V. Kuzmiak, A. A. Maradudin, "Localized defect modes in a two-dimensional triangular photonic crystal," Phys. Rev. B 57, pp. 15242-15250, 1998.

9. W. D. Zhou, J. Sabarinathan, P. Bhattacharya, B. Kochman, E. W. Berg, P. C. Yu, and S. W. Pang, "Characteristics of a Photonic Bandgap Single Defect Microcavity Electroluminescent Device," IEEE Journal of Quantum Electron. 37, pp. 1153-1160, 2001.

10. R. K. Lee, O. Painter, B. Kitzke, A. Scherer, and A. Yariv, "Emission properties of a defect cavity in a two dimensional photonic bandgap crystal slab," J. Opt. Soc. Am. B 17, pp. 629-633, 2000.

11. H. Y. Ryu, J. K. Hwang, and Y. H. Lee, "The Smallest Possible Whispering-Gallery-Like Mode in the Square Lattice Photonic-Crystal Slab Single-Defect Cavity," IEEE Journal of Quantum Electron. 39, pp. 314-322, 2003. 
12. K. Srinivasan and O. Painter, "Momentum space design of high-Q photonic crystal optical cavities,"Optics Express 10, pp. 670-684, 2002.

13. T. Yoshie, J. Vuckovic, M. Loncar, A. Scherer, H. Chen, D. Deppe, "Optical characterization of high quality two dimensional photonic crystal cavities," Lasers and Electro-Optics, 2002. CLEO '02. Technical Digest 1, pp. 191, 2002.

14. O. Painter, R. K. Lee, A. Yariv, A. Scherer, J. D. O’Brien, P.D. Dapkus, I. Kim, “Two-dimensional Photonic Crystal Defect Laser," Science 284, pp. 1819-1821, 1999.

15. S. Fan, P. R. Villeneuve, J. D. Joannopoulos, and H. A. Haus, "Channel Drop Tunneling through Localized States," Phys. Rev. Lett. 80, pp. 960-963, 1998.

16. C. Jin, S. Han, X. Meng, B. Cheng, and D. Zhang, "Demultiplexer using directly resonant tunneling between point defects and waveguides in a photonic crystal," J. Appl. Phys. 91, pp. 4771-4773, 2002.

17. C. Manolatou, M. J. Khan, S. Fan, P. R. Villeneuve, H. A. Haus, J. D, Joannopoulos, "Coupling of modes analysis of resonant channel add-drop filters," IEEE Journal of Quantum Electron. 35, pp. 1322-1331, 1999.

18. M. Imada, S. Noda, A. Chutinan, M. Mochizuki, and T. Tanaka, "Channel Drop Filter Using a Single Defect in a 2D Photonic Crystal Slab Waveguide," IEEE Journal of Lightwave Technol. 20, pp. 845-850, 2002.

19. J. Vuc;kovic, M. Loncar, H. Mabuchi, and A. Scherer, "Design of photonic crystal microcavities for cavity QED," Phys. Rev. B 65, pp. 016608 (1) - 016608(11), 2001.

20. Y. Xu, Y. Li, R. K. Lee, and A. Yariv, "Scattering-theory analysis of waveguide-resonator coupling," Phys. Rev. E 62, pp. 7389-7404, 2000.

21. A. Adibi, Y. Xu, R. K. Lee, A. Yariv, A. Scherer, "Properties of the slab modes in photonic crystal optical waveguides," IEEE Journal of Lightwave Technol. 18, pp. 1554-1564, 2000.

22. K. S. Yee, "Numerical solution of initial boundary value problems involving Maxwell's equations in isotropic media," IEEE Trans. Antennas Propagat.AP-14, pp. 302-307, 1966.

23. J. P. Berenger, "A perfectly matched layer for the absorption of electromagnetic waves," J. Comput. Phys. 114, pp. 185-200, 1994.

24. D. E. Merewether, R. Fisher, and F. W. Smith, "On implementing a numeric Huygen's source scheme in a finite difference program to illuminate scattering bodies," IEEE Trans. Nucl. Sci. NS-27, p. 1829, 1980.

25. R. Collin, Foundations for Microwave Engineering, IEEE Press, $2^{\text {nd }}$ Edition, New Jersey, 2001. 